\title{
Bilateral ocular melanocytosis with malignant melanoma of the choroid
}

\author{
JOHN R. GONDER, ${ }^{1}$ JERRY A. SHIELDS, ${ }^{2}$ JEFFREY L. SHAKIN, ${ }^{2}$ AND \\ DANIEL M. ALBERT ${ }^{1}$
}

From the ${ }^{2}$ Oncology Service at Wills Eye Hospital and Research Institute,

Thomas Jefferson University, Philadelphia, and the 'Harvard Medical School,

Department of Ophthalmology, Massachusetts Eye and Ear Infirmary, Boston,

Massachusetts, USA

SUMMARY A woman with bilateral ocular melanocytosis developed a malignant melanoma of the choroid in one eye. The ocular melanotic hyperpigmentation, present since childhood, clinically involved the conjunctiva and episcleral and uveal tract of both eyes. To our knowledge this is only the second reported case of a uveal malignant melanoma in a patient with bilateral ocular melanocytosis. There is evidence that patients with ocular or oculodermal melanocytosis have an increased incidence of malignant melanoma of the uvea. It is possible that patients with bilateral ocular melanocytosis have a greater chance of developing a uveal malignant melanoma than do those with unilateral involvement.

Oculodermal melanocytosis (naevus fuscocoeruleus ophthalmomaxillaris), a melanocytic hyperpigmentation of the globe and periorbital skin, was described in 1939 by Ota and Tanino. ${ }^{1}$ Melanocytic hyperpigmentation involving the globe alone is called ocular melanocytosis, and similar hyperpigmentation involving the skin alone is called dermal melanocytosis. Malignant melanoma of the uveal tract has been reported to occur in patients with ocular or oculodermal melanocytosis. ${ }^{2-4}$ Malignant melanoma of the uveal tract has also been described in cases of bilateral ocular melanocytosis ${ }^{3}$ and bilateral oculodermal melanocytosis. ${ }^{2}$ Herein we report a case of malignant melanoma of the uveal tract in a person with bilateral ocular melanocytosis.

\section{Case report}

A 74-year-old white woman was referred to the Oncology Service of Wills Eye Hospital for assessment of a tumour of the right eye. She had noted painless loss of vision about 6 weeks prior to presentation. The patient had been aware of episcleral pigmentation in both eyes since early childhood. The family history was unremarkable.

Correspondence to J. A. Shields, MD, Oncology Service, Wills Eye Hospital, 9th and Walnut Streets, Philadelphia, PA 19107, USA.
CLINICAL EXAMINATION

The patient's visual acuity with correction was $6 / 7 \cdot 5$ and $6 / 5$ in the right and left eyes respectively. External examination revealed bilateral ocular melanocytosis (Fig. 1). No periocular pigmentation was noted. The

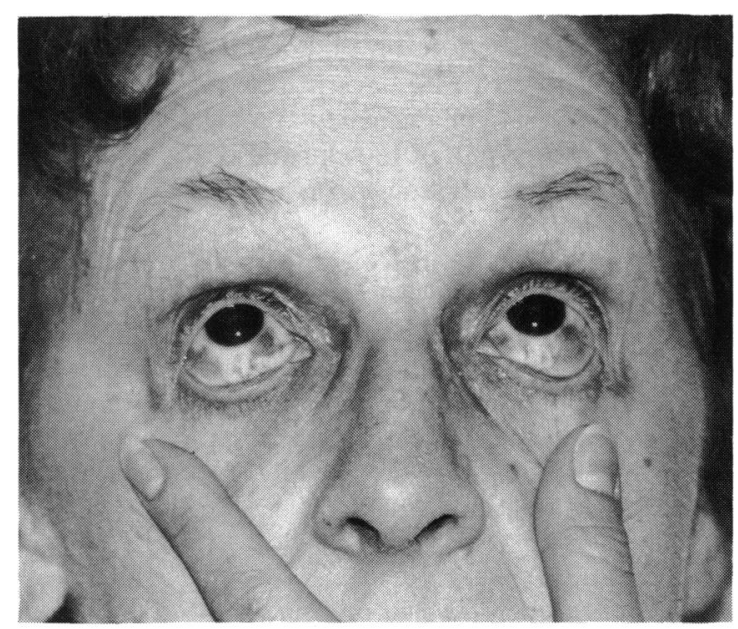

Fig. 1 External photograph of the patient showing the bilateral ocular melanocytosis. Note the pigmentation inferiorly on both globes. No dermal pigmentation was noted. 
Fig. 2 A high-power photomicrograph of the malignant melanoma demonstrating the epithelioid malignant melanoma cells. (Haematoxylin and eosin, $\times 560$ ).

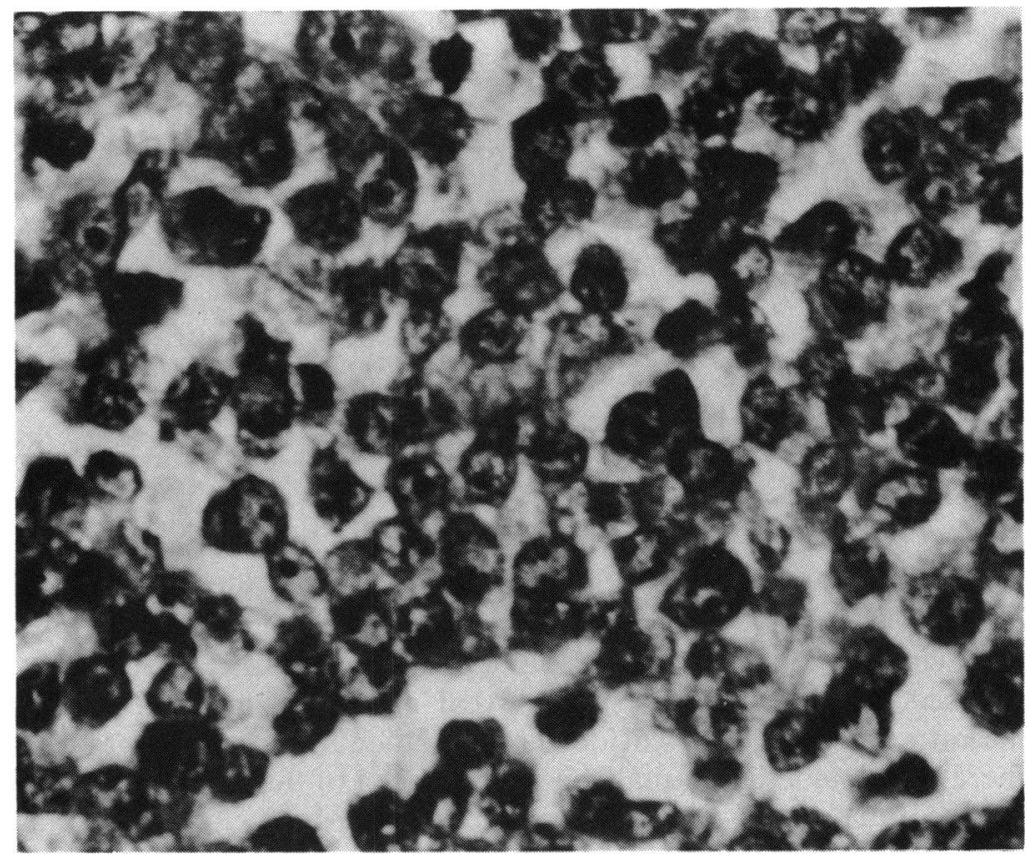

intraocular pressure by applanation was $12 \mathrm{mmHg}$ in both eyes. Slit-lamp biomicroscopy revealed bilateral conjunctival and episcleral pigmentation. The conjunctiva in the lower fornix of each eye contained small nodular pigmented thickenings.

Funduscopic examination of the right eye showed a large pigmented choroidal mass clinically estimated to be $18 \times 15 \times 8 \mathrm{~mm}$. A dependent serous retinal detachment was associated with the mass. The fundus of the left eye showed drusen and reticular pigmentary degeneration of the midperiphery. Both fundi were noted to be dark in colour. The clinical diagnoses were (1) bilateral ocular melanocytosis; (2) malignant melanoma of the choroid of the right eye; and (3) bilateral nodular pigmented lesions of the conjunctiva.

The findings on fluorescein angiography and ultrasonography were consistent with the diagnosis of malignant melanoma of the choroid of the right eye. At operation an incisional ${ }^{32} \mathrm{P}$ test was positive at $173 \%$. The tumour-containing right eye was removed by the 'no touch' cryoenucleation technique. In addition 3 pigmented conjunctival nodules were submitted for histological study.

\section{GROSS PATHOLOGY}

The enucleated right eye measured $24 \times 25 \times 24 \mathrm{~mm}$ with $8 \mathrm{~mm}$ of optic nerve attached. Examination of the intact globe revealed patches of episcleral pigmentation most marked in the equatorial region. On opening the globe we noted that the retina was detached inferotemporally by a bilobed solid choroidal mass measuring $15 \times 15 \times 8 \mathrm{~mm}$.

Microscopic examination showed that the tumour was composed almost completely of epithelioid malignant melanoma cells (Fig. 2). It was confined to the choroid, with no rupture of Bruch's membrane or extrascleral extension. The overlying retinal pigment epithelium showed disruption, with drusen formation. The remainder of the choroid contained an increased number of densely pigmented melanocytes. Remote from the tumour was a discrete choroidal nodule composed of pigmented cells with spindleshaped nuclei. The iris and ciliary body contained heavy pigmentation but were otherwise unremarkable. A serous retinal detachment was present. Episcleral pigmented dendritic melanocytes were noted at the limbus and at the equator. The optic nerve appeared normal.

The conjunctival specimens contained increased numbers of hyperpigmented dendritic melanocytes in the substantia propria (Fig. 3). In one section the dendritic melanocytes extended deep to the substantia propria to involve the orbital fat.

The final histopathological diagnosis was malignant melanoma of the choroid, epithelioid cell type, associated with ocular melanocytosis.

\section{Discussion}

In patients with ocular and oculodermal melanocytosis the pigmentation may involve the conjunctiva, 


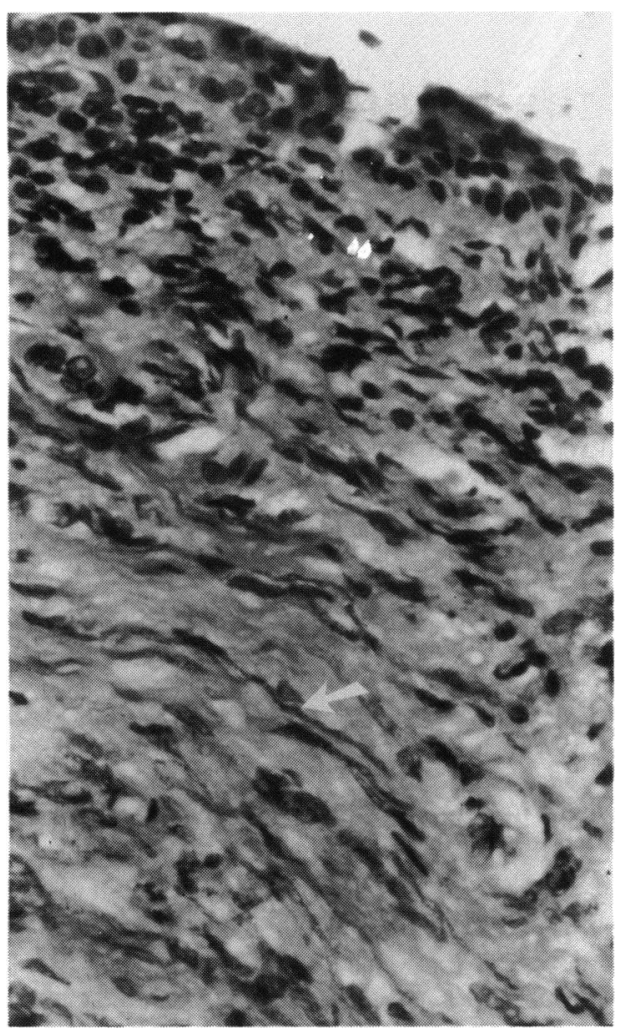

Fig. 3 Photomicrograph of the nodular conjunctival lesion showing the pigmented dendritic melanocytes in the substantia propria (arrow). Inflammatory cells are present in the upper substantia propria. (Haematoxylin and eosin, $\times 347)$.

episclera, and uveal tract, as illustrated by our case. An unusual aspect of our case was that both the conjunctiva and choroid had areas of nodular thickening by the hyperpigmented melanocytes. Other tissues that had been reported to be involved in ocular or oculodermal melanocytosis included the optic disc, the skin of the eyelid, temple, forehead, and cheek, the orbit, meninges, tympanic membrane, the orbital and cranial bones, the pharynx, nasal mucosa, and hard palate. ${ }^{5}$ Oculodermal melanocytosis is more common in oriental ${ }^{2}$ than white per- sons and 4 times more prevalent in women than men. The pigmentation is congenital in $60 \%$ of persons, while others developed the pigmentation at puberty or during pregnancy. ${ }^{5}$

In addition to choroidal malignant melanoma ocular and oculodermal melanocytosis have been associated with melanomas of the iris, orbit, ${ }^{6}$ central nervous system, ${ }^{7}$ and skin. ${ }^{8}$ On the basis of accumulated reports in the literature ocular and oculodermal melanocytosis have been stated to have an increased malignant potential. Blodi ${ }^{9}$ recently reported a case in which a malignant melanoma of the choroid developed in the uninvolved eye in a patient with ocular melanocytosis to support his opinion that patients with this condition may not be predisposed to develop uveal malignant melanoma. Further studies are necessary to resolve this controversy, with the definitive answer lying in a prospective study.

Dr Gonder was a fellow in ophthalmic pathology at Harvard Medical School and is now a fellow in vitreoretinal diseases in the Retina Service at Wills Eye Hospital.

This study was supported in part by the R. Samuel McLaughlin Foundation. National Eye Institute Grant EY01917. The Retina Research and Development Foundation, Philadelphia, the Pennsylvania Lions Sight Conservation and Eye Research Foundation, and the Ocular Oncology Fund. Wills Eye Hospital.

\section{References}

1 Ota M. Tanino $\mathrm{H}$. Nevus fusco-coeruleus ophthalmomaxillaris. Tokvo Med J 1939; 63: 1243-4.

2 Halasa A. Malignant melanoma in a case of bilateral nevus of ota. Arch Ophthalmol 1970; 84: 176-8.

3 Makley TA, King CM. Malignant melanoma in melanosis oculi. Trans Am Acad Ophthalmol Otolaryngol 1967; 77: 638-41.

4 Yanoff M, Zimmerman LE. Histogenesis of malignant melanoma of the uvea; the relationship of congenital ocular melanocytosis and neurofibromatosis to uveal melanomas. Arch Ophthalmol 1967; 77: 331-6.

5 Estima A, Carneiro $R$. Ota naevus: presentation of a case. $B r J$ Plast Surg 1972; 25: 49-52.

6 Jay B. Malignant melanoma of the orbit in a case of oculoderma melanosis. Br J Ophthalmol 1965; 49: 359-63.

7 Sang DN, Albert DM, Sober AJ, McMeekin TO. Nevus of ota with contralateral cerebral melanoma. Arch Ophthalmol 1977; 95: $1820-4$.

8 Dorsey CS, Montgomery $\mathrm{H}$. Blue nevus and its distinction from Mongolian spot and nevus of ota. J Invest Dermatol 1954; 22: 225-36.

9 Blodi FC. Ocular melanocytosis and melanoma. Am J Ophthalmol 1975; 80: 389. 